9

each fluores-

cent spot is composed of a single mRNA and several nascent chains

TRANSLATION

\title{
Live stream: translation at single-mRNA resolution
}

Translation dynamics in living cells is poorly understood, because a method for directly imaging the translation of single mRNA molecules in real time was lacking. Now, in Science and Cell, four research groups report the development of such a method to track single mRNAs and its application to the study of translation dynamics in different cellular contexts.

The four groups devised, independently, a system that couples live fluorescent imaging of mRNAs with that of translated nascent peptide chains. They constructed expression vectors encoding the mRNAs of interest engineered for doublelabelling: their 3 ' untranslated region contains bacteriophage-originated hairpin-forming repeats, to which matching fluorescent proteins bind, thereby labelling the mRNA; and the $5^{\prime}$ end of the coding sequence encodes a peptide that is stably bound by antibody fragments, which are fluorescently labelled or fused to fluorescent proteins, thereby labelling nascent chains. Three groups engineered the 5 ' end to encode an array of SunTag epitopes, whereas Morisaki et al. used an array of FLAG tags.

When the expression vectors were introduced into human cell lines, bright, two-coloured fluorescent spots were visualized in the cells. The researchers showed that the emerging nascent tag peptides were rapidly bound by the antibody fragments. The addition of puromycin, which releases nascent chains from ribosomes, caused the loss of peptide but not mRNA labelling from the spots, demonstrating that the

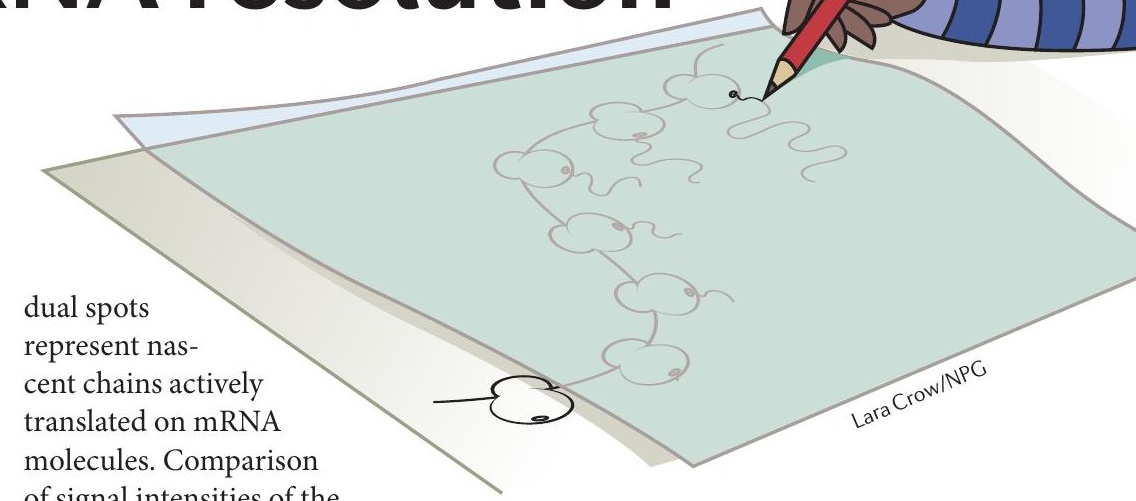

of signal intensities of the

labelled hairpin repeats and tag arrays revealed that each fluorescent spot is composed of a single mRNA and several nascent chains, thus providing an effective means of visualizing polysomes.

Different approaches were used to measure translation elongation rates by each group, but the resulting calculated rates were similar - all within a range of $\sim 3-10$ amino acids per second, which is consistent with rates previously obtained in mammalian cells by ribosome profiling (5.6 amino acids per second). These are the first measurements of translation elongation rates of single mRNA molecules to be obtained in live cells.

Wu et al. and Wang et al. used the method to investigate locally restricted translation in neurons and found that, contrary to the hypothesis that mRNAs being transported to specific sites of translation are translationally inactive, transported mRNAs were actively translated during transport. Yan et al. showed that mRNA isoforms can exhibit considerable heterogeneity in translation efficiency; they, as well as Wu et al., found that mRNAs rapidly alternated between translating and non-translating states. Morisaki et al. demonstrated that the addition to the system of the HA tag and matching antibody fragments with a different fluorescent dye can support three-colour imaging in cells, revealing that different polysomes mostly do not interact. This could come in handy for imaging regulatory factors together with mRNAs and nascent peptides.

In summary, this imaging method is the first to enable tracking of the translation kinetics of individual, engineered mRNA molecules in living cells. Further development will be required to enable tracking of the translation of single endogenous mRNAs.

Eytan Zlotorynski quantification of single RNA translation dynamics in living cells. Science http://dx.doi.org/10.1126/ science.aaf0899 (2016) | Wu, B. et al. Translation dynamics of single mRNAs in live cells and neurons. Science http://dx.doi.org/10.1126/ science.aaf1084 (2016) | Yan, X. et al. Dynamics of translation of single mRNA molecules in vivo. Cell 165, 976-989 (2016) | Wang, C. et al. Real-time imaging of translation on single mRNA transcripts in live cells. Cell 165, 990-1001 (2016) FURTHER READING Buxbaum, A. R., Haimovich, G. \& Singer, R. H. In the right place at the right time: visualizing and understanding mRNA localization. Nat. Rev. Mol. Cell Biol. 16, 95-109 (2015)
ORIGINAL ARTICLES Morisaki, T. et al. Real-time 